\title{
SYNTACTIC PHYLOGENETIC TREES
}

\author{
KEVIN SHU, SHARJEEL AZIZ, VY-LUAN HUYNH, DAVID WARRICK, MATILDE MARCOLLI
}

\begin{abstract}
In light of recent controversies surrounding the use of computational methods for the reconstruction of phylogenetic trees of language families (especially the Indo-European family), a possible approach based on syntactic information, complementing other linguistic methods, appeared as a promising possibility, largely developed in recent years in Longobardi's Parametric Comparison Method. In this paper we identify several serious problems that arise in the use of syntactic data from the SSWL database for the purpose of computational phylogenetic reconstruction. We show that the most naive approach fails to produce reliable linguistic phylogenetic trees. We identify some of the sources of the observed problems and we discuss how they may be, at least partly, corrected by using additional information, such as prior subdivision into language families and subfamilies, and a better use of the information about ancient languages. We also describe how the use of phylogenetic algebraic geometry can help in estimating to what extent the probability distribution at the leaves of the phylogenetic tree obtained from the SSWL data can be considered reliable, by testing it on phylogenetic trees established by other forms of linguistic analysis. In simple examples, we find that, after restricting to smaller language subfamilies and considering only those SSWL parameters that are fully mapped for the whole subfamily, the SSWL data match extremely well reliable phylogenetic trees, according to the evaluation of phylogenetic invariants. This is a promising sign for the use of SSWL data for linguistic phylogenetics. We also argue how dependencies and nontrivial geometry/topology in the space of syntactic parameters would have to be taken into consideration in phylogenetic reconstructions based on syntactic data. A more detailed analysis of syntactic phylogenetic trees and their algebro-geometric invariants will appear elsewhere.
\end{abstract}

\section{INTRODUCTION}

This paper is based on a talk given by the last author at the workshop "Phylogenetic Models: Linguistics, Computation, and Biology" organized by Robert Berwick at the CSAIL department of MIT in May 2016.

The reconstruction of phylogenetic trees of language families is a crucial problem in the field of Historical Linguistics. The construction of an accurate family tree for the Indo-European languages accompanied and originally motivated the development of Historical Linguistics, and has been a focus of attention for linguists for the span of two centuries. In recent years, Historical Linguistics has seen a new influx of mathematical and computational methods, originally developed in the context of mathematical biology to deal with species phylogenetic trees, see for instance [5], [10], [12], [28], 23], [34]. A considerable amount of controversy arose recently in relation to the accuracy and effectiveness of these methods and the related problem of phylogenetic inference. In particular, claims regarding the phylogenetic tree of the Indo-European languages made in [6] were variously criticized by historical linguists, see the detailed discussion in [27].

Most of the literature dealing with computational phylogenetic trees in the context of Linguistics focused on the use of lexical data, in the form of Swadesh lists of words, and the encoding as binary data of the counting of cognate words, see for instance the articles in [12]. Other reconstructions used phonetic data and sound change, as in [5], or a combination of several types of linguistic data (referred to as "characters"), including phonetic, lexical, and morphological properties, as in 
[3], 34]. A different approach to linguistic phylogenetic reconstruction, based on syntactic parameters, was developed recently in [17], [18], [19], [20, [21]. This method is known as Parametric Comparison Method (PCM). A coding theory perspective on the PCM was given in [22].

The notion of syntactic parameters arises in Generative Linguistics, within the Principles and Parameters model developed by Chomsky in [7], [8]. A more expository account of syntactic parameters is given in [2]. Syntactic parameters are conceived as binary variables that express syntactic features of natural languages. The notion of syntactic parameters has undergone changes, reflecting changes in the modeling of generative grammar: for a recent overview of the parametric modeling of morphosyntactic features see [30]. A main open problems in the parameteric approach for comparative generative grammar is understanding the space of syntactic parameters, identifying dependence relations between parameters and possibly identifying a fundamental set of such variables that would represent a good system of coordinates for the space of languages. Recently, the use of mathematical methods for the study of the space of syntactic parameters of world languages was proposed in [26], 29], 32].

At present, the only available extensive database of binary parameters describing syntactic features is the SSWL database [35], which collects data of 115 parameters over 253 world languages. It is debatable whether the binary variables collected in SSWL represent fundamental syntactic parameters: surface orders, for instance, are often confounded with the deep underlying parameter values. Moreover, SSWL does not record any dependence relations between parameters. Different data of syntactic parameters have been used in [20], [21], with dependence relations taken into account, and more data are being collected by these authors and will hopefully be available soon. For the purpose of this paper, we will use the terminology "syntatic parameters" loosely for any collection of binary variables describing syntactic features of natural languages. We work with the SSWL data, simply because it is presently the most extensive database available of syntactic structures.

In Section 2 of this paper we show that just using the Hamming distance between vectors of binary variables extracted from the SSWL data and the Neighborhood-Joining Method for phylogenetic inference gives very poor results as far as linguistic phylogenetic trees are concerned. We identify several different sources of problems, some inherent to the SSWL data, some to the inference methodology, and some more generally related to the use of syntactic parameters for phylogenetic linguistics.

In the Section 4 we review the method of Phylogenetic Algebraic Geometry of [24] and the main results of [1] and [33] on phylogenetic ideals and phylogenetic invariants that we need for applications to the analysis of syntactic phylogenetic trees. In Section 5 we show how one can use techniques from Phylogenetic Algebraic Geometry to test the reliability of syntactic parameter data for phylogenetic linguistics, by using known phylogenetic trees that are considered reliable, and to test the reliability of candidate phylogenetic trees assuming a certain degree of reliability of the syntactic data.

In Section 6 we argue that dependencies between the syntactic variables recorded in the SSWL database should be taken into consideration in order to improve the reliability of these data for phylogenetic reconstruction. In particular, the presence of geometry/topology in this set of data and the presence of different degrees of recoverability of some of the SSWL syntactic variables in Kanerva network tests indicate that an appropriated weighted use of the data that accounts for these phenomena may improve the results.

Acknowledgment. The first author is supported by a Summer Undergraduate Research Fellowship at Caltech. Part of this work was performed as part of the activities of the last author's 
Mathematical and Computational Linguistics lab and CS101/Ma191 class at Caltech. The last author is partially supported by NSF grants DMS-1201512 and PHY-1205440.

\section{PHYLIP ANALYSIS OF SSWL}

We discuss here the problems that occurs in a naive analysis of the SSWL database using the phylogenetic tree algorithm PHYLIP. We identify the main types of errors that occur and the possible sources of the problems. We will discuss in $\$ 4$ how one can eliminate some of the problems and obtain more accurate phylogenetic trees from SSWL data, using different methods.

2.1. Data and Code. We acquired the syntactic language data from the SSWL database with two different methods, one consisting of downloading the data as a .csv file directly, with the results separated in the format "language|property|value", and one achieved by scraping the data into a .json file, formatted as a list of lists of binary variables, in the format "language' : \{ 'parameters' : 'values'\}". This was done with a python script data_obtainer.py which went through all of SSWL and dumped the data as desired.

The SSWL data, stored in a more convenient . json file format produced by the first author, are available as the file full_langs.json which can be downloaded at the URL address http://www.its.caltech.edu/ matilde/PhylogeneticSSWL2.

We created, for each language in the database, a vector of binary variables representing the syntactic traits of that language as recorded in the SSWL database, with value 1 indicated that the language possessed the respective trait, and value 0 indicating that the language does not possess the trait.

One of the main sources of problems regarding the use of SSWL data arises already at this stage: not all languages in the database have all the same parameters mapped. The lack of information about a certain number of parameters for certain languages alters the counting of the Hamming distances, as it requires a choice of normalization of the string length, with additional entries added representing lack of information. This clearly generates problems, as this inconsistency generates mistakes in the counting of Hamming distances and in the tree reconstruction. In \$2.2 we will illustrate specific examples where this problem occurs.

The Hamming distance algorithm HF . py takes two equal-length binary sequences, throwing an error if this length requirement is violated, and returns the sum of all bitwise XORs between them, or the total number of differences. In this way, we construct with distance_matrix_checker.py the Hamming distance matrix $M_{a b}=d_{H}\left(\ell_{a}, \ell_{b}\right)$, whose entries are the Hamming distances between the vectors of binary syntactic parameters of languages $\ell_{a}$ and $\ell_{b}$.

For example, Germanic languages on average have normalized Hamming distance in the range 0.3-0.4. Old Saxon and Old English have a Hamming distance of 0.17 from German, while Swiss German has distance 0.09. Modern English has below average differences at 0.27. While these distances may appear reasonable, one can detect easily another major source of problems in the use of SSWL data for phylogenetic reconstruction. Many languages belonging to very different families have small Hamming distance: for example, the Indo-European Hindi (60\% mapped in SSWL) and the Sino-Tibetan Mandarin (87\% mapped in SSWL) receive a normalized distance of 0.12 . This is certainly in large part due to the different level of accuracy with which the two languages are mapped in the same database. However, one can also observe syntactic similarities between languages belonging to different families, which are not due to poor recording of the respective data, but are a genuine consequence of the syntactic properties being described. 
This $253 \times 253$ matrix of Hamming distances was then given as input to the PHYLIP packag€ $\biguplus^{1}$ for phylogenetic tree reconstruction, which is widely used in Mathematical Biology. Given the Hamming distance matrix $M_{a b}=d_{H}\left(\ell_{a}, \ell_{b}\right)$, the PHYLIP software provides several options for tree construction from distance matrix data: additive tree model, ultrametric model, neighbor joining method, and average linkage clustering (UPGMA). The resulting tree produced by PHYLIP, containing all 253 languages in the SSWL database, is contained in outfile, where the tree in the text file is drawn with dashes and exclamation points. The information of the output tree and distances is also given in the output file outtree in Newick format, with parentheses and commas. The accompanying file key.txt contains the key that indicates the full language name that corresponds to each two-letter string in outfile. The output files can be opened in any text editor.

The python code and the output files, prepared by the second, third and fourth authors of this paper, are available at http://www.its.caltech.edu/ matilde/PhylogeneticSSWL

2.2. Main problems in the resulting tree. A quick inspection of the output file obtained by running PHYLIP on the SSWL data immediately reveals that there are many problems with the resulting phylogenetic tree. We will give explicit examples here that illustrate some of the main type of problems one encounters. There are many more such examples one can easily find by inspecting the output tree available in the repository at the URL indicated above.

2.3. Sources of problems. An important problem in computational phylogenetic reconstruction is how to validate statistically the model. There are well known problem inherent in using the Hamming distance as a source for phylogenetic trees. Estimating tree branch lengths is a hard problem. Distance matrices can be non-additive due to error, and it is typically difficult to distinguish distances that deviate from additivity due to change from deviations due to error. This problem is significant even in the context of Biology, where the use of DNA data is more reliable than the use of vectors of binary variables coming from linguistic properties. For a discussion of some of these issues in Biology see [9]. For a comparison of phylogenetic methods (not including syntactic parameters) in Linguistics, see [3].

As we discuss with individual specific examples in the subsections that follow, there are several different source of problems that combine to create different kinds of errors in the resulting phylogenetic tree. The main problems are the following:

(1) inherent problems in the computational method based on Hamming distances, as discussed above;

(2) problems with non-uniform coverage of syntactic data across different languages and language families in the SSWL database;

(3) the nature of the syntactic variables recorded in the SSWL database (for instance with respect to surface versus deep structure) and the presence of relations between these variables;

(4) the existence of languages belonging to unrelated linguistic families that can be similar at the level of syntactic structures.

Clearly, some of these problems are of linguistic nature, like the last one listed, while others are of computational nature, like the first one, while others depend on the nature and accuracy of the SSWL data. It is difficult to disentangle the effects of each individual problem on the output tree, but the examples listed below illustrate cases where one can identify one of the problems listed here as the most likely origin of the mistakes one sees in the resulting phylogenetic tree.

${ }_{1}^{1}$ http://evolution.genetics.washington.edu/phylip/software.html 


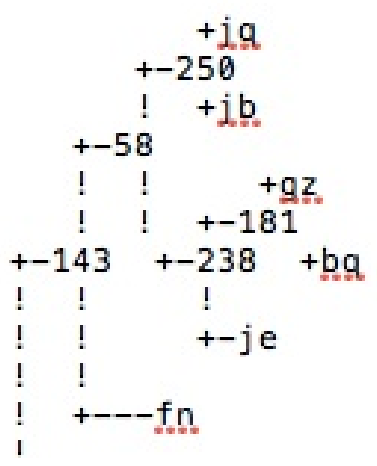

Figure 1. PHYLIP output subtree of Latin languages: jq=Spanish, jb=Catalan, $\mathrm{gz}=$ Sicilian, bq=Italian, je=Portuguese, $\mathrm{fn}=$ French.

2.3.1. Misplacement of languages within the correct subfamily tree. This type of problem occurs when a group of languages are correctly identified as belonging to the same subfamily of a given historical-linguistic family, but the internal structure of the subfamily tree appears inconsistent with the structure generally agreed upon based on other linguistic data.

In the naive PHYLIP analysis of the SSWL database we see an example of this kind by considering the subtree of the Latin languages within the Indo-European family. The shape of this subtree, as it appears in in the output file, is of the form illustrated in Figure 1. We see here that, although these languages are correctly grouped together as belonging to the same subfamily, the relative position within the subtree does not agree with what historical linguistic methods have established. Indeed, one can easily see, for instance, that the position of Portuguese in the subtree is incorrectly placed closer to Italian and Sicilian, than to Spanish and Catalan. This example is interesting because the error does not appear to be due to the poor mapping of parameters for these languages: Italian and Sicilian are 100\% mapped in SSWL and Spanish, Catalan, and Portuguese are $84 \%$ mapped. So these are among some of the best recorded languages in the database, and still their respective position in the phylogenetic tree does not agree with reliable reconstructions from Historical Linguistics. It is interesting to compare the reconstruction obtained in this way, with the one obtained, on a different set of syntactic data, by Longobardi's Parametric Comparison in [20], which has Italian and French as a pair of two nearby branches, and Spanish and Portuguese as another pair of nearby branches. This example appears to outline an issue arising from the way syntactic variables are classified in the SSWL (as opposed to the different list of syntactic parameters used in [20]). We discuss in $\$ 6$ below some of the problems of dependencies between the SSWL syntactic variables that may be at the sources of this kind of problem.

2.3.2. Placement of languages in the wrong subfamily tree. Another type of mistake one finds in the naive phylogenetic tree reconstruction from SSWL syntactic data is illustrated by the Germanic languages in Figure 2. In this case, we find that most of the languages in this subtree are correctly grouped together as Germanic, but a language that clearly belongs to a different subfamily is also placed in the same group. It is very puzzling why Ancient Neapolitan ends up incorporated in the tree of Germanic languages rather then near Italian and the other dialects of Italian in the subtree of Latin languages of Figure 1. Linguistically, one could perhaps argue that Ancient Neapolitan did in fact have several Germanic influences due to the Ostrogoths, but it is more reasonable to expect such influences to appear at the lexical rather than syntactic level. Moreover, the specific placement within the Germanic tree near Faroese, Norwegian and Icelandic, does not necessarily 


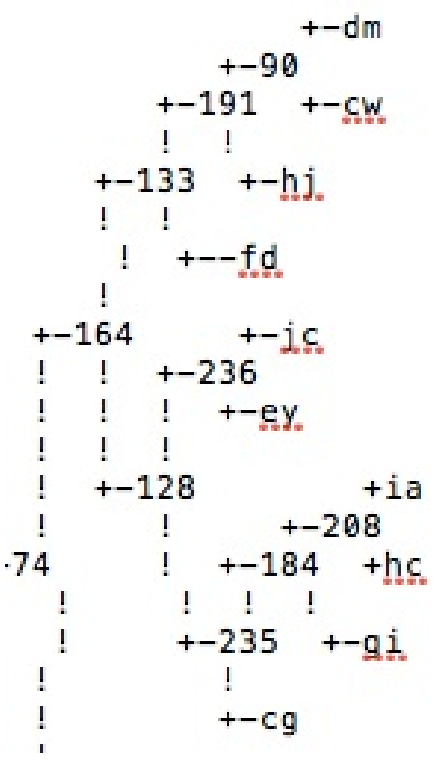

FiguRE 2. PHYLIP output subtree of Germanic languages: $d m=$ Norwegian, $\mathrm{cw}=$ Faroese, $\mathrm{hj}=$ Italian Ancient Neapolitan, $\mathrm{fd}=$ Icelandic, $\mathrm{jc}=$ Afrikaans, $\mathrm{ey}=$ West Flemish, ia=Dutch, hc=German, gi=Swedish, $\mathrm{cg}=$ English .

reflect this hypothesis. In terms of the accuracy with which these languages are recorded in the SSWL database, Ancient Neapolitan is $83 \%$ mapped, while its nearest neighbor on this PHYLIP output tree have Norwegian, which is also mapped with a similar accuracy of $84 \%$, and Faroese and Icelandic with a lower accuracy of $69 \%$. It is possible that this example already reflects a problem with the different accuracy of mapping of different languages in the SSWL database, or it may be a problem with the algorithmic reconstruction method itself. There are several similar instances in the output tree, which point to a problem that is systematic, hence likely generated by the method of phylogenetic reconstruction adopted in this naive analysis.

2.3.3. Proximity of languages from unrelated families. Another type of problem that occurs frequently in the output tree of this naive analysis is the case of completely unrelated languages (from completely different language families) that are placed in adjacent positions in the tree. We see an example in Figure 3, where the Mayan K'iche' language and Georgian (Kartvelian family) are placed next to each other in the tree. Both K'iche' and Georgian are 69\% mapped in the SSWL database. Although this is not as accurate a mapping as some of the languages we discussed in the previous examples, it is nonetheless the same level of precision available, for instance, for some of the Germanic languages in the previous example, which were at least placed correctly in the Germanic subtree. Thus, the type of problem we see in this example is not entirely due to poor mapping of the languages involved. It must be also an effect of other factors like the computational reconstruction method used, as in the previous class of examples. However, there can also be some purely linguistic factors involved. Namely, there are known cases of languages belonging to unrelated historical linguistic families that may appear close at the syntactic level. This type of phenomenon may be responsible for at least part of the cases where one finds unrelated languages placed in close proximity in the output tree. This is an indication that one should not rely on syntactic data alone, without accompanying them with other linguistic data, that can provide, for example, a prior subdivision of languages into language families. Using the same method of phylogenetic tree reconstruction on data already grouped into linguistic families, with individual family 


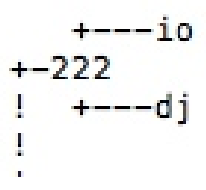

Figure 3. Misplaced proximity: io=K'iche', dj=Georgian.

trees separately constructed, improves the accuracy of the resulting trees. Other combinations of syntactic and lexical/morphological data can be used to improve accuracy.

2.3.4. The position of ancient languages in the tree. Finally, there is an additional problem one encounters in the naive phylogenetic reconstruction based on the SSWL data, namely the position of the ancient languages in the tree. Clearly, the algorithm assumes that all the data correspond to leaves of the tree and that the inner nodes are hidden variables, while the fact that we do have knowledge of some of the ancient languages and that several are recorded in the SSWL database means that some of the inner nodes should in fact carry some of the data. This problem can be resolved if the inner languages would be placed as a single leaf attached to the corresponding inner node. By inspecting the resulting output tree we see that sometimes this is the case, and the inner node to which the corresponding ancient language is attached reasonably with respect to the modern languages that derived from it. One such example is the position of Old English with respect to the tree of the Germanic languages in Figure 4. However, in other cases, ancient languages are correctly placed in proximity of each other, but in the wrong position, in the tree, with respect to the resulting modern languages. This is the case with Ancient Greek and Latin (see Figure 5). In this case, the algorithm correctly captures the close syntactic proximity between Ancient Greek and Latin, but it does not place these two languages correctly with respect to either the tree of Latin languages nor the modern part of the Hellenic branch. This problem can be improved by first subdividing the data into language families and smaller subfamilies and then perform the phylogenetic tree reconstruction on the subfamilies separately, so that the corresponding ancient language is placed correctly, and then related the resulting trees by proximity of the ancient languages. However, this method clearly applies only where enough other linguistic information is available, in addition to the syntactic data. It should be noted, moreover, that, while Ancient Greek is correctly placed in proximity to Latin, Homeric Greek is entirely misplaced in the PHYLIP tree reconstruction and does not appear in proximity of the Ancient Greek of the classical period, even though both Homeric and Ancient Greek are mapped with the best possible accuracy (100\% mapped) in the SSWL database.

2.4. The Indo-European tree. Although the many problems illustrated above render a phylogenetic reconstruction based solely on SSWL data unreliable, it is still worth commenting on what one obtains with this method regarding some of the controversial early branchings of the IndoEuropean tree. Again, the same type of systematic problems illustrated above occur repeatedly when one analyzes these regions of the output tree.

For example, Tocharian A and B are treated by the PHYLIP reconstruction as modern languages leaves of the tree and placed in immediate proximity of Hittite and in close proximity of some of the modern Indo-Iranic languages, like Pashto and Punjabi, and a further step away from some Turkic languages like Tuvan. The proximity of Tocharian and Hittite suggests here a Tocharian-Anatolian 


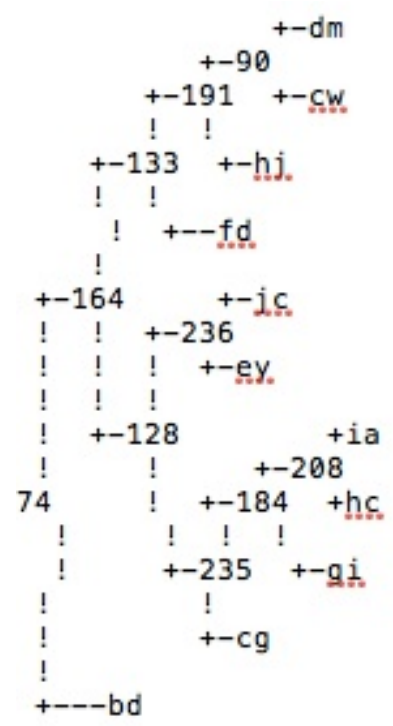

Figure 4. The position of Old English with respect to the Germanic languages: $\mathrm{bd}=$ Old English.

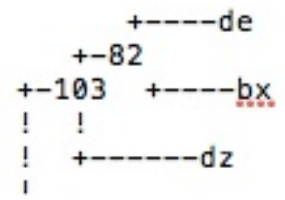

Figure 5. Proximity of Ancient Greek and Latin: de=Latin, bx=Ancient Greek, $\mathrm{dz}=$ Medieval Greek.

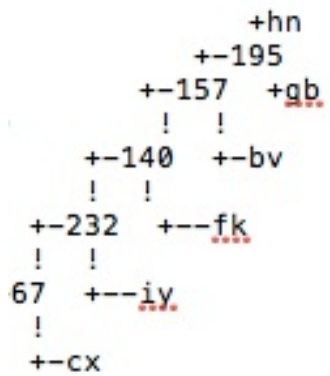

Figure 6. Tocharian-Anatolian branching: $\mathrm{gb}=$ Tocharian $\mathrm{A}$, $\mathrm{hn}=$ Tocharian $\mathrm{B}$, $\mathrm{bv}=$ Hittite, $\mathrm{fk}=$ Pashto, iy=Panjabi, $\mathrm{cx}=$ Tuvan (Turkic).

branching. The placement of the Indo-Iranic languages in proximity of this Tocharian-Anatolian branching is likely arising from the fact that the Indo-Iranic branch of the Indo-European family is very poorly mapped in the SSWL database, with the ancient languages entirely missing and very few of the modern languages recorded, hence the reconstructed tree necessarily skips over all these missing data. The complete absence of Sanskrit from the current version of the SSWL database (the entry in the database is just an empty place holder) in particular causes the phylogenetic reconstruction to miss entirely the proximity of the Indo-Iranic and the Hellenic branches. Near the subtree shown in Figure 6 one finds several instances of misplaced languages of the type discussed in $\$ 2.3 .3$ above. 


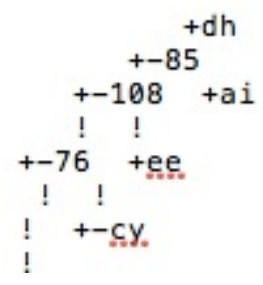

Figure 7. Eastern Armenian: cy = Eastern Armenian (84\%), ee = Pima (misplaced Uto-Aztecan), ai = Ossetic Digor, dh= Ossetic Iron.

The situation with the Armenian branch is very problematic in the PHYLIP analysis of the SSWL data. There are three entries recorded in the database: Western Armenian is 68\% mapped, while Eastern Armenian appears as two different entries in the database, one 84\% mapped and the other only $52 \%$ mapped. Classical Armenian only appears as an empty place holder with no data in the current version of the database. These three data points are not placed in proximity of one another in the PHYLIP reconstruction. Western Armenian ends up completely misplaced (it appears in proximity of Korean and Japanese). This misplacement may be corrected if one first subdivides data by language families and then runs the phylogenetic reconstruction only on the Indo-European data. The better mapped entry for Eastern Armenian is placed in proximity of the subtree of Figure 6 containing the Tocharian-Anatolian branch and some Indo-Iranian languages (plus some other misplaced languages from other families). The nearest neighbors that appear in this region of the tree are Digor Ossetic and Iron Ossetic: again this is likely an effect of the poor mapping of the Indo-Iranic branch of the Indo-European family, as in the case of Figure6. Another error due to misplacement from an entirely different family occurs, with the Uto-Aztecan Pima placed in this same subtree, see Figure 7. This subtree is placed adjacent to a subtree containing a group of Balto-Slavic languages (and some misplaced languages) with both of these branches then connecting to the subtree of Figure 6. The poorly mapped Eastern Armenian entry (52\%) is placed as single leaf attached to an otherwise deep inner node of the tree. Another language that is often difficult to position in the Indo-European tree, Albanian (68\% mapped), is misplaced in the PHYLIP reconstruction, and placed next to Gulf Arabic (69\% mapped).

These examples confirm the fact that a naive phylogenetic analysis of the SSWL database cannot deliver any reliable information on the question of the early branchings of the Indo-European tree.

\section{Phylogenetic Networks}

We verified that the same types of problems illustrated in the previous subsections occur when the SSWL data are analyzed using phylogenetic networks instead of the PHYLIP phylogenetic trees.

We compiled the SSWL data [35], using only the Indo-European languages, which have more complete parameter information as a sample set. As in the tree analysis discussed before, we input the syntactic parameters as a sequence of binary strings into the phylogenetic networks programs.

The Splitstree 4 program ${ }^{2}$ generated a split tree, which is intuitively a confidence interval on trees. The farther from 'tree-like' the generated tree, the less any given tree is able to describe the evolution of the languages. The output of this program indicated that the phylogenetics of

${ }^{2}$ http://ab.inf.uni-tuebingen.de/data/software/splitstree4/download/manual.pdf 


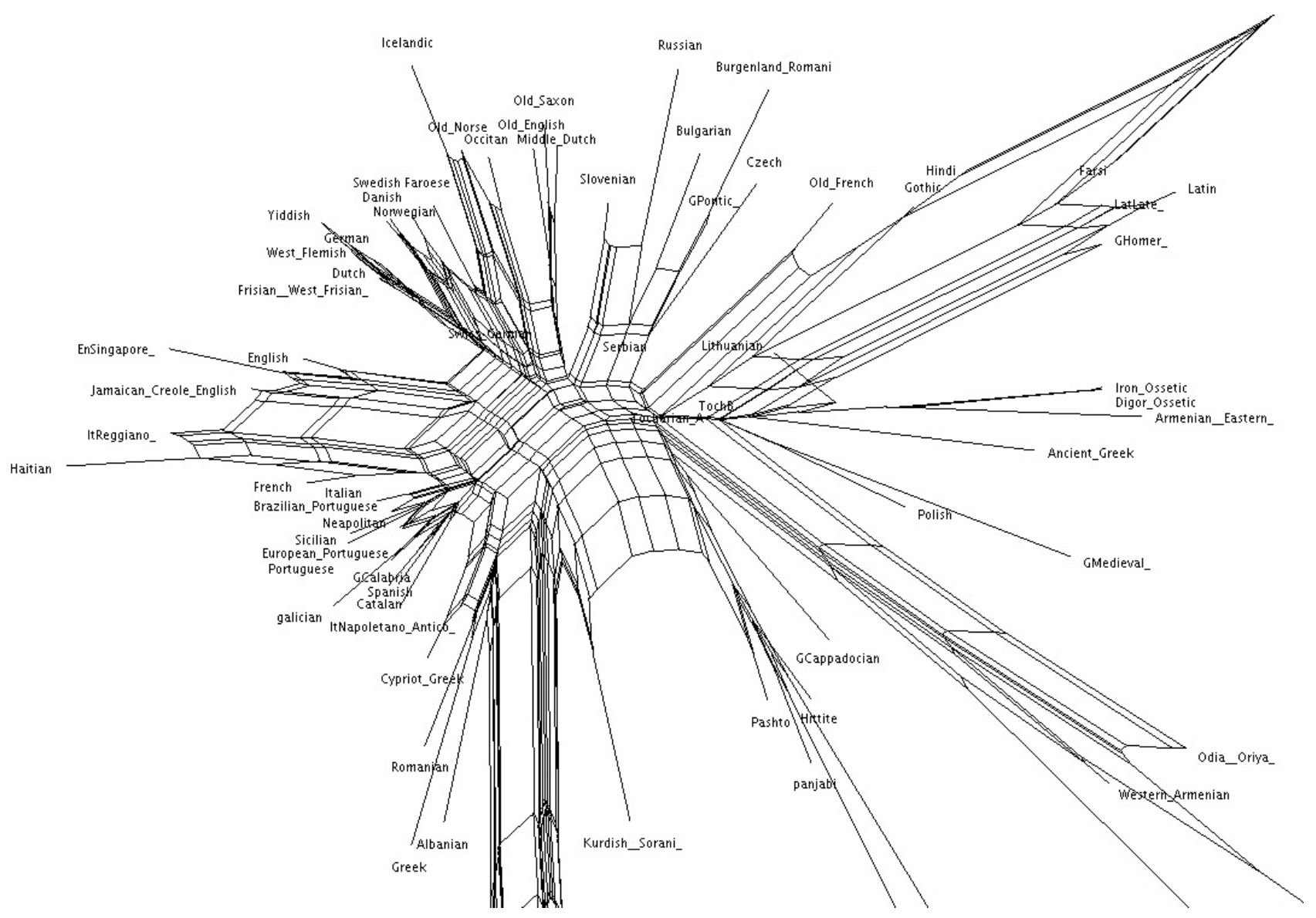

Figure 8. Phylogenetic network produced by Splittree 4 on the entire SSWL database.

languages analyzed on the basis of SSWL syntactic parameters diverges strongly from being treelike. As discussed before, this may be regarded as further indication of systematic problems that create high uncertainties in the candidate trees. These are again an illustration of the effect of a combination of the factors (1)-(4) listed in $\$ 2.3$.

We also fed the same data to the Network 5 program ${ }^{3}$ This generated a phylogenetic network, using the median-joining algorithm which represents all of the shortest-path length (maximum parsimony) trees which are possible given the data.

We discuss below some of the aspects of the network generated by Splittree 4 in comparison to some of the outputs described above obtained with the PHYLIP phylogenetic trees. Figure 8 illustrates a large region of the phylogenetic network produced by Splittree 4 using the entire set of SSWL data. It is evident that some of the same problems we have discussed before occur in this case as well, in particular the misplacement of the ancient languages with respect to their modern descendent (see the position of Latin and Ancient Greek, for example). However, with respect to the PHYLIP results discussed above, we see less instances of languages that get completely misplaced within the wrong family. For example, as one can see from Figures 10 and 9, Ancient Neapolitan now appears correctly placed in the Latin languages (and near Spanish) rather than misplaced among the Germanic languages as in Figure2. However, one can see that other problems that occurred in the PHYLIP reconstructions for this group of languages are still present in the

3 http://www.fluxus-engineering.com/Network5000_user_guide.pdf 


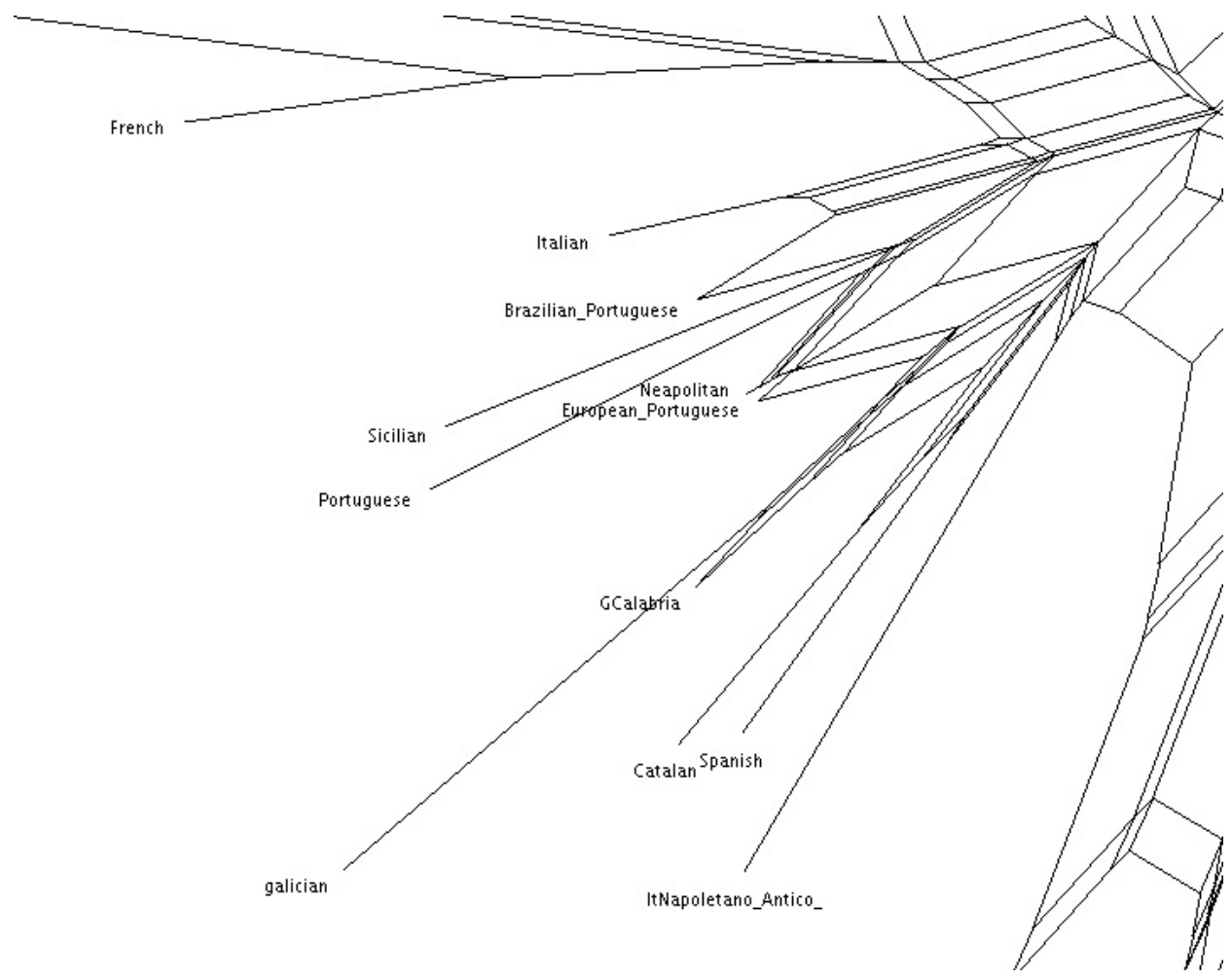

FIGURE 9. Latin languages region of the phylogenetic network.

Splittree 4 network. For example, as in Figure 1, Portuguese appears closer to Italian than to Spanish in the network of Figure 9, contrary to the general understanding of the phylogenetic tree of the Latin languages. (We will discuss the case of the subtree of the Latin languages more in detail in $\$$ below.) Misplacements of languages within these smaller subfamilies are still occurring, however: one can see that, for example, in the positioning of the Romance language Occitan in the region of the phylogenetic network in proximity of Germanic languages like Old Norse and Icelandic in Figure 10 .

The results of the Splittree 4 phylogenetic networks analysis of the Indo-European languages are available as the file Indo_Euro.nex, which can be downloaded at the URL

http://www.its.caltech.edu/ matilde/PhylogeneticSSWL2

\section{Phylogenetic Algebraic Geometry}

Given the unsatisfactory results one obtains in analyzing the SSWL database with software aimed at phylogenetic reconstructions, one can turn the problem on its head and try to obtain specific quantitative estimates of the level of reliability or unreliability of specific subsets of the SSWL data for the purpose of phylogenetic, by relying on existing reconstructions of linguistic phylogenetic trees, obtained by other linguistic methods and other sources of data, which are considered reliable reconstructions. The problem is then to test the distribution at the leaves of the tree obtained from the SSWL data with specific polynomial invariants associated to a given reliable tree. Such invariants would be vanishing on a probability distribution at the leaves obtained from an evolutionary process modeled by a Markov model on the tree, hence we can use 


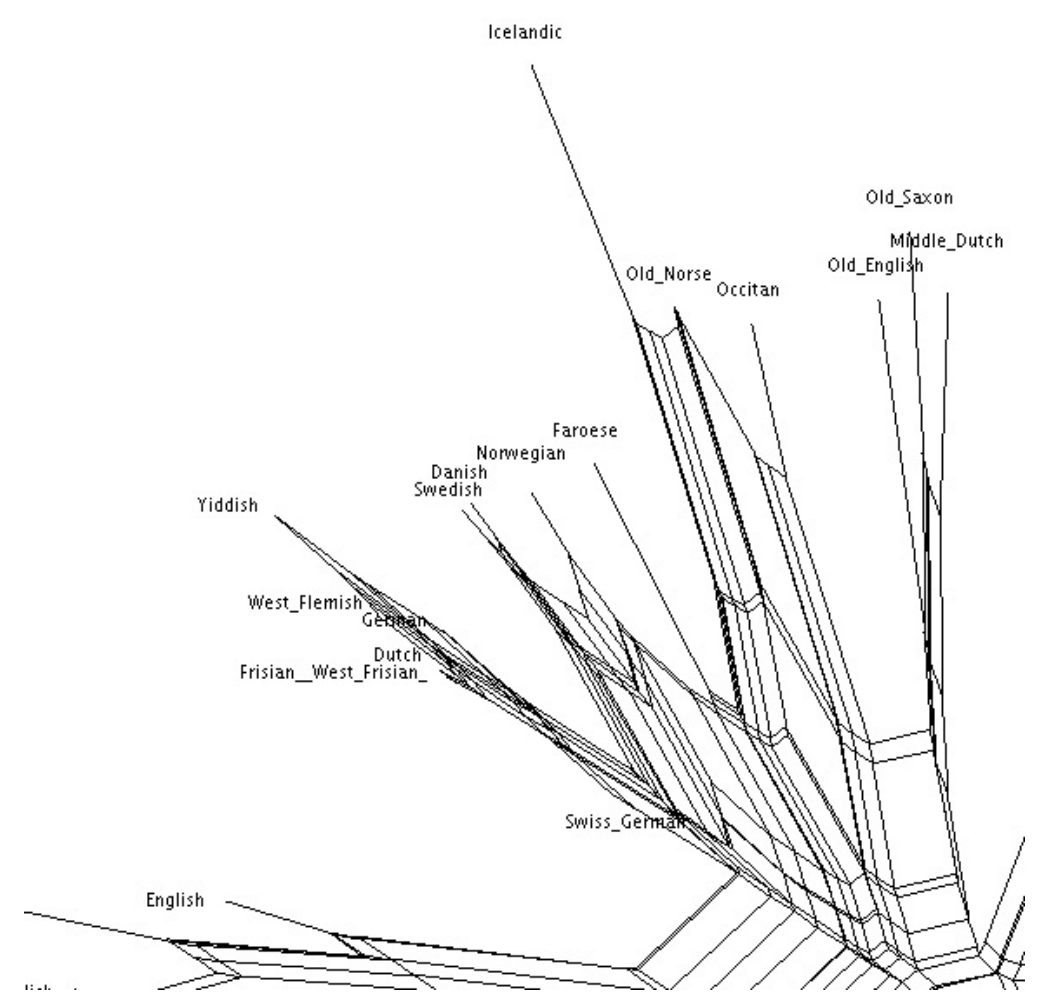

FiguRE 10. Germanic languages region of the phylogenetic network.

the estimate of how far the values are from zero as a numerical estimate of a degree of unreliability of the data for phylogenetic reconstruction. Again, this does not identify explicitly the source of the problem, among the various possible causes outlined above, but it still gives a numerical estimate that can be useful in trying to improve the results. We propose here to use methods from phylogenetic algebraic geometry to achieve this goal. We first give a quick review of the main setting of phylogenetic algebraic geometry and then we illustrate in some specific examples how we intend to use these techniques for the purpose described here.

4.1. Phylogenetic models: general assumptions. The basic setup for linguistic phylogenetic models consists of a dynamical process of language change (which in our case means change of syntactic parameters), considered as a Markov process on a binary tree (a finite tree with all internal vertices of valence 3).

It can be argued whether trees really give the best account of language change based on syntactic data, rather than more general non-simply-connected graphs (generally referred to as "networks"). We will return to discuss some empirical reasons in favor of phylogenetic networks instead of trees in $\$ 6$ below. The mathematics of phylogenetic networks is discussed at length in [14] and [15]. About the use of phylogenetic networks in Linguistics, see [23].

Another general assumption of phylogenetic models, which requires careful examination in the case of applications to Linguistics, is the usual assumption that the variables (binary variables in the case of syntactic parameters) behave like independent identically distributed variables, whose dynamics evolves according to the same Markov process. This assumption is especially problematic when dealing with syntactic parameters because of the presence of relations between parameters that are not entirely understood, so that it is currently extremely hard to ensure one is using a set of independent binary variables. Moreover, while acceptable in first approximation, even 
the assumption that the underlying Markov model driving the change should be the same for all syntactic parameters appears problematic. The fact that different syntactic parameters have very different frequencies of occurrence among world languages certainly suggests otherwise. We will return to this point in $\$ 6$ and suggest a possible approach, based on the results of [26], to correct, at least in part, for this problem.

The leaves of the tree correspond to the modern languages with observed values of the parameters giving a joint probability distribution

$$
\mathbb{P}\left(X_{\ell_{1}}=i_{1}, \ldots, X_{\ell_{n}}=i_{n}\right)=p_{i_{1}, \ldots, i_{n}},
$$

with $i_{k} \in\{0,1\}$, and with $n$ the number of leaves. Here the quantity $p_{i_{1}, \ldots, i_{n}}$ represents the frequency with which syntactic parameters of the languages $\ell_{1}, \ldots, \ell_{n}$ at the leaves of the tree have values $\left(i_{1}, \ldots, i_{n}\right) \in\{0,1\}^{n}$, respectively.

In the usual setting of Markov models for phylogenetic reconstructions, one further assumes that all the inner nodes are hidden variables and that only the distribution at the leaves of the tree is known. Here again we encounter a problem with respect to applications to Linguistics. In certain language families, like the Indo-European family, several ancient languages have known parameters. In the SSWL database, for instance, Ancient Greek is one of the very few languages that are $100 \%$ mapped with respect to their list of 115 parameters. Thus, one needs to consider some of the inner vertices as known rather than hidden. One way to do that is to consider a single leaf coming out of some of the inner vertices that will correspond to the known values of the parameters at that vertex. As we discussed in $\$ 2.2$ above, one encounters problems with the placement of the ancient languages in the PHYLIP reconstruction of the syntactic phylogenetic trees, which should be corrected for. Better results are obtained when one first separates out the data into language families and subfamilies and builds trees for smaller subfamilies first, including the known data about the ancient languages, and then combines these trees into a larger tree. This procedure avoids the type of problem mentioned in $\$ 2.2$, by which the greater syntactic similarity between some of the ancient Indo-European languages like Latin and Ancient Greek is detected correctly, but in turn prevents their respective placement in the correct position with respect to the modern languages that originated from them.

For a given set of $n$ leaves, there are

$$
\tau_{n}=\frac{(2 n-4) !}{(n-2) ! 2^{n-2}}
$$

different possible binary tree topologies. Clearly, it is not a computationally efficient strategy to analyze all of them. However, one would like to have some computable invariants that one can associate to a given candidate tree $T$, which estimates how accurate $T$ is as a phylogenetic tree, among all the $\tau_{n}$ possible choices, given knowledge of the joint probability distribution (4.1) at the leaves. The Phylogenetic Algebraic Geometry approach (see [24], 25] and the survey [4]) aims at constructing such phylogenetic invariants using Algebraic Geometry and Commutative Algebra. We review the main ideas in the next subsection.

4.2. Phylogenetic varieties and ideals. We consider here the Jukes-Cantor model describing a Markov process on a binary rooted tree $T$ with $n$ leaves. The stochastic behavior of the model is determined by the datum of a probability distribution $(\pi, 1-\pi)$ at the root vertex (the frequency of expression of the 0 and 1 values of the syntactic parameters at the root) and the datum of a bistochastic matrix

$$
M^{e}=\left(\begin{array}{cc}
1-p_{e} & p_{e} \\
p_{e} & 1-p_{e}
\end{array}\right)
$$


along each edge of the tree. These data $\left(\pi, M^{e}\right)$ are often referred to in the literature as parameters of the model. In order to avoid confusion with our use of the term parameter for the syntactic binary variables, we will refer to the $\left(\pi, M^{e}\right)$ as "stochastic parameters". For a tree $T$ with $n$ leaves, and variables with $k$ states, the number of stochastic parameters is

$$
N=(2 n-3) k(k-1)+k-1 \text {. }
$$

In our case, with binary variables, we have $k=2$ and the number of stochastic parameters of the model is simply $N=4 n-5$.

Phylogenetic invariants are polynomial functions $\phi$ that vanish on all the expected distributions $p_{i_{n}, \ldots, i_{n}}$ at the tails of the tree $T$, for all values of the stochastic parameters $\left(\pi, M^{e}\right)$.

The simplest example of such an invariant is the linear polynomial

$$
\phi\left(z_{i_{n}, \ldots, i_{n}}\right)=-1+\sum_{i_{n}, \ldots, i_{n}} z_{i_{n}, \ldots, i_{n}},
$$

since the joint distribution at the leaves is normalized by $\sum_{i_{n}, \ldots, i_{n}} p_{i_{n}, \ldots, i_{n}}=1$. This invariant is uninteresting, in the sense that it is independent of the tree $T$, hence it does not provide any information about distinguishing between candidate phylogenetic trees. In general one seeks other, more interesting, phylogenetic invariants $\phi_{T}$, and the minimum number of such invariants required for phylogenetic inference. An answer to this question is provided by Algebraic Geometry, as shown in [1], [24], [25], [33].

Consider the polynomial ring $\mathbb{C}\left[z_{i_{1}, \ldots, i_{n}}\right]$, where $n$ is the number of leaves of the tree and and $i_{k} \in$ $\{0,1\}$ for all $k=1, \ldots, n$. The phylogenetic invariants are defined by the vanishing $\phi_{T}\left(p_{i_{1}, \ldots, i_{n}}\right)=0$. This condition determines an ideal $\mathcal{I}_{T}$ in the polynomial ring. For a Markov model as above, with $N=4 n-5$ stochastic parameters $\left(\pi, M^{e}\right)$, one obtains a polynomial map

$$
\Phi: \mathbb{C}^{4 n-5} \rightarrow \mathbb{C}^{2^{n}}
$$

that assigns $\Phi\left(\pi, M^{e}\right)=p_{i_{1}, \ldots, i_{n}}$. This is, more explicitly, of the form

$$
p_{i_{1}, \ldots, i_{n}}=\Phi\left(\pi, M^{e}\right)=\sum_{w_{v} \in\{0,1\}} \pi_{w_{v_{r}}} \prod_{e} M_{w_{s(e)}, w_{t(e)}}^{e},
$$

with a sum over "histories" (paths in the tree) consistent with the data at the leaves. This determines an algebraic variety, the phylogenetic variety, given by the Zariski closure

$$
V_{T}=\overline{\Phi\left(\mathbb{C}^{4 n-5}\right)} \subset \mathbb{C}^{2^{n}}
$$

Dually we have a map

$$
\Psi: \mathbb{C}\left[z_{i_{1}, \ldots, i_{n}}\right] \rightarrow \mathbb{C}\left[x_{1}, \ldots, x_{4 n-5}\right]
$$

with $\operatorname{Ker} \Psi=\mathcal{I}_{T}$, where $\mathcal{I}_{T}$ is the phylogenetic ideal.

One can use phylogenetic invariants to select between candidate phylogenetic trees in the following way. Suppose one obtains, through some phylogenetic algorithm, a candidate phylogenetic tree $T$. One also has available the joint probability distribution (4.1) of the binary variables at the leaves. By evaluating phylogenetic invariants $\phi_{T} \in \mathcal{I}_{T}$ at the observed distribution $p_{i_{n}, \ldots, i_{n}}$, one can check whether the candidate tree $T$ satisfies

$$
\left|\phi_{T}\left(p_{i_{n}, \ldots, i_{n}}\right)\right|<\epsilon
$$

for all phylogenetic invariants $\phi_{T} \in \mathcal{I}_{T}$, and for a fixed error size $\epsilon$. The candidate tree $T$ is an acceptable phylogenetic tree if and only if the estimate (4.2) is satisfied. Geometrically, the test 4.2 can be rephrased as the property that the point $p_{i_{1}, \ldots, i_{n}} \in \mathbb{C}^{2^{n}}$ is $\epsilon$-close to the phylogenetic variety $V_{T}$ if and only if $T$ is an acceptable phylogenetic tree. Computationally, this method requires obtaining a set of explicit generators for the phylogenetic ideal $\mathcal{I}_{T}$. 
In the case of the Jukes-Cantor model with $k=2$, it was proved in [33] that the phylogenetic ideal $\mathcal{I}_{T}$ is generated by polynomials of degree two. A completely explicit set of generators for the Jukes-Cantor model with $k=2$ was obtained in [1], where it is proved that phylogenetic ideal $\mathcal{I}_{T}$ generated by the $3 \times 3$-minors of all edge flattenings of the tensor $P=\left(p_{i_{1}, \ldots, i_{n}}\right)$. The edge flattenings are defined by the following procedure. Start with a tree $T$ with Markov model $\left(\pi, M^{e}\right)$ and with $P \in \mathbb{C}^{2^{n}}$ the joint probability distribution $P=\left(p_{i_{1}, \ldots, i_{n}}\right)$ at the $n$ leaves. The choice of an edge $e$ in a tree $T$ with $n$ leaves determines two connected components of $T \backslash\{e\}$, hence two sets of leaves $\left\{\ell_{1}, \ldots, \ell_{r}\right\}$ and $\left\{\ell_{r+1}, \ldots, \ell_{n}\right\}$. Thus, the $2^{n}$ binary variables at the $n$ leaves are partitioned into a set of $2^{r}$ variables and a set of $2^{n-r}$ variables, and the joint distribution $P=\left(p_{i_{1}, \ldots, i_{n}}\right)$ determines a $2^{r} \times 2^{n-r}$-matrix Flat $_{e, T}(P)$ specified by setting

$$
\text { Flat }_{e, T}(P)(u, v)=P\left(u_{1}, \ldots, u_{r}, v_{1}, \ldots, v_{n-r}\right) .
$$

It can be shown that the rank of this matrix is $\operatorname{rank}\left(\operatorname{Flat}_{e, T}(P)\right) \leq 2$ (for binary variables, $k=2$ ), hence all $3 \times 3$ minors of the matrix must vanish. It is shown in [1] that, for $k=2$ any number $n$ of leaves, the phylogenetic ideal $\mathcal{I}_{T}$ is generated by the $3 \times 3$ minors of the matrices Flat $_{e, T}(P)$ of all edge flattenings. It is easy to see that, even for small trees, there is a very large number of these $3 \times 3$ minors, hence the number of generators of the phylogenetic ideal grows rapidly with the size of the tree.

Note that, while for the purpose of validating a candidate phylogenetic tree $T$ it would be necessary to check that all these generators of the phylogenetic ideal vanish (or nearly vanish as in (4.1)), in order to invalidate a candidate tree it sufficed to find at least one of these $3 \times 3$ minors for one of the flattenings that evaluates on the observed joint distribution $P=\left(p_{i_{1}, \ldots, i_{n}}\right)$ to a value larger than the allowed error size $\epsilon$.

\section{Phylogenetic invariants and syntactic trees}

In this section we show how phylogenetic invariants can be used to improve the phylogenetic tree reconstructions based on SSWL syntactic data.

5.1. Phylogenetic invariants of small syntactic trees. We focus here on sufficiently small subtrees of the syntactic phylogenetic tree of languages compiled from the SSWL data, for which the computation of phylogenetic invariants becomes feasible. Using phylogenetic invariants, we compare the small trees obtained in this way with phylogenetic trees obtained by other linguistic methods and considered reliable, so as to estimate the validity of the joint distribution at the leaves obtained from SSWL data.

We present here an example, based on the subtree of the Latin languages within the IndoEuropean family. A more detailed analysis of other subtrees of the Indo-European family will be presented elsewhere.

We have seen in $\$ 2.3 .1$ that the naive PHYLIP analysis of the entire SSWL database misplaces Portuguese in the subtree of the Indo-European languages that collects the Latin languages. We have also seen in 2.3.4 that the same analysis misplaces Latin, separating it from the tree of the modern languages that originated from it.

We now perform a more accurate analysis, still using only the SSWL data, but where we use the a priori knowledge of the grouping of certain languages into a subfamily. Thus, we select only the languages Latin, Italian, French, Spanish, Portuguese.

The phylogenetic tree that is generally agreed, through other linguistic reconstructions, to best represent the relative position of these languages would be a tree topology as illustrated in Figure 11. Note that this is also the tree reconstruction for this group of languages obtained in [20] using a set of syntactic parameters different from those recorded in the SSWL database. 


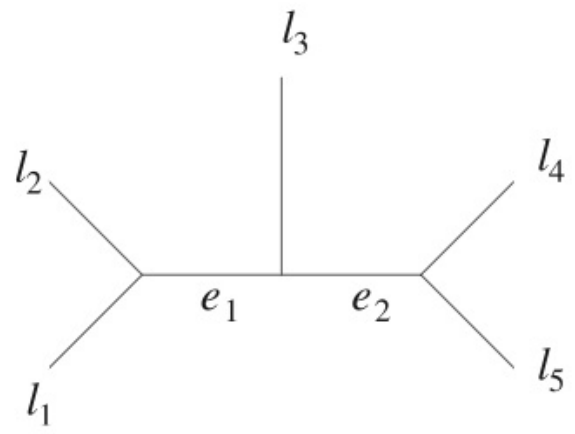

Figure 11. Tree topology for the phylogenetic tree of the Latin languages, with $\ell_{1}=$ French, $\ell_{2}=$ Italian, $\ell_{3}=$ Latin, $\ell_{4}=$ Spanish, $\ell_{5}=$ Portuguese.

The tree of Figure 11 has two possible splits: $\left\{\ell_{1}, \ell_{2}\right\} \cup\left\{\ell_{3}, \ell_{4}, \ell_{5}\right\}$ and $\left\{\ell_{1}, \ell_{2}, \ell_{3}\right\} \cup\left\{\ell_{4}, \ell_{5}\right\}$. The corresponding flattenings are given by the matrices

$$
\text { Flat }_{e_{1}}(P)=\left(\begin{array}{llllllll}
p_{00000} & p_{00001} & p_{00010} & p_{00011} & p_{00100} & p_{00101} & p_{00110} & p_{00111} \\
p_{1000} & p_{01001} & p_{01010} & p_{01011} & p_{01100} & p_{01101} & p_{01110} & p_{01111} \\
p_{10000} & p_{10001} & p_{10010} & p_{10011} & p_{10100} & p_{10101} & p_{10110} & p_{10111} \\
p_{11000} & p_{11001} & p_{11010} & p_{11011} & p_{11100} & p_{11101} & p_{11110} & p_{1111}
\end{array}\right) \quad \text { Flat }_{e_{2}}(P)=\left(\begin{array}{llll}
p_{00000} & p_{00001} & p_{00010} & p_{00011} \\
p_{0100} & p_{00101} & p_{00110} & p_{00111} \\
p_{01000} & p_{01001} & p_{01010} & p_{01011} \\
p_{01100} & p_{01101} & p_{01110} & p_{01111} \\
p_{10000} & p_{10001} & p_{10010} & p_{10011} \\
p_{10100} & p_{10101} & p_{10110} & p_{10111} \\
p_{11000} & p_{11001} & p_{11010} & p_{11011} \\
p_{11100} & p_{11101} & p_{11110} & p_{1111}
\end{array}\right)
$$

where the $p_{i_{1}, i_{2}, i_{3}, i_{4}, i_{5}}$ are the frequencies of the observed binary variables at the ends, under the assumption that these behave like independent equally distributed random variables, evolving according to the same Markov model on the tree.

Using the data of SSWL parameters for these five languages reported in the Appendix, we

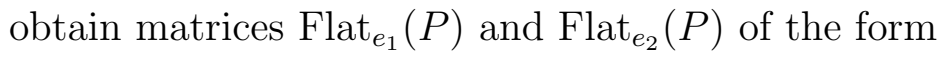

$$
\begin{gathered}
\text { Flat }_{e_{1}}(P)=\left(\begin{array}{cccccccc}
\frac{31}{106} & \frac{1}{106} & \frac{1}{106} & 0 & \frac{23}{106} & \frac{3}{106} & 0 & \frac{1}{53} \\
\frac{1}{106} & 0 & 0 & \frac{1}{106} & 0 & \frac{1}{106} & 0 & \frac{3}{106} \\
\frac{5}{106} & 0 & \frac{1}{53} & 0 & 0 & 0 & 0 & 0 \\
\frac{1}{53} & 0 & \frac{1}{106} & \frac{4}{53} & 0 & 0 & 0 & \frac{21}{106}
\end{array}\right) \\
\text { Flat }_{e_{2}}(P)=\left(\begin{array}{cccccc}
\frac{31}{106} & \frac{1}{106} & \frac{1}{106} & 0 & 0 \\
\frac{23}{106} & \frac{3}{106} & 0 & \frac{1}{53} & 0 \\
\frac{1}{106} & 0 & 0 & \frac{1}{106} & 0 \\
0 & \frac{1}{106} & 0 & \frac{3}{106} & 0 \\
\frac{5}{106} & 0 & \frac{1}{53} & 0 & 0 \\
0 & 0 & 0 & 0 & 0 \\
\frac{1}{53} & 0 & \frac{1}{106} & \frac{4}{53} & 0 \\
0 & 0 & 0 & \frac{21}{106} & 0
\end{array}\right)
\end{gathered}
$$

Evaluating all the $3 \times 3$ minors of these matrices with Maple and selecting the maximum absolute value of the resulting phylogenetic invariants gives

$$
\max \left|\phi_{T}\left(p_{i_{1}, \ldots, i_{5}}\right)\right|=\frac{2415}{1191016}=0.0020277 .
$$


The fact that for the tree of Figure 11 the distribution at the leaves determined by the SSWL parameters is extremely close to being a zero of all the phylogenetic invariants implies that the SSWL parameters are in fact in very good agreement with the recognized correct topology of the phylogenetic tree, but only when the set of languages is previously restricted to a smaller subfamily and only the SSWL parameters that are fully mapped for that subfamily are taken into account.

This result seems to indicate that the main source of the problems we encounter when doing a naive phylogenetic analysis using the entire SSWL database are not necessarily due to an intrinsic problem with the SSWL data (that is, it is not primarily due to problem number (3) in the list in \$2.3). It seems rather that the problems encountered above stem from a combination of problems (1), (2), and (4). The use of the phylogenetic invariants method bypasses problem (1), while the prior restriction to a smaller subfamily bypasses problems (2) and (4). A more detailed analysis of this approach with phylogenetic invariants, applied to other language subfamilies using SSWL data will be carried out more extensively elsewhere.

\section{Dependencies And Geometry}

As we already mentioned above, the problem of the construction of reliable syntactic phylogenetic trees is closely related to the problem of relations and dependencies between syntactic parameters. Are there universal relations that hold across all languages? Are there relations that depend on language families? Can these relations be expressed geometrically, as is the case with relations between continuous coordinates that give rise to topological or differentiable manifolds? Are there different geometries associated to different language families? How detectable are relations between syntactic parameters computationally? Recently, a mathematical approach to these questions was proposed in [22], [26], 29], 32].

In [29], it was shown, again using SSWL data, that syntactic parameters of different language families have different persistent homology. The persistent generators of $H_{0}$ appear to correspond to a subdivisions of a given language family into major subfamilies, such as, for example, the Indo-Iranic and the European subfamilies of the Indo-European family, or the Mande, AtlanticCongo, and Kordofanian subfamilies of the Niger-Congo family. A persistent generator of the $H_{1}$ was found in the case of the Indo-European family. It appears to be related to the position of the Hellenic branch in the Indo-European family. It is presently unclear whether this reflects the effect of a genuine historical-linguistic phenomenon, such as an influence of Ancient Greek, at the syntactic level, upon some other European languages (such as some of the Slavic languages), whether it detects the presence of homeoplasy in syntactic parameters, or whether it is due to the nature and format of the syntactic data collected in the SSWL database. However, the presence of non-trivial persistent generators of the $H_{1}$ in the persistent homology of the data set is a strong indicator that networks (non-simply-connected graphs) and not trees may provide a better topology for syntactic phylogenetic linguistics.

In [26], it was shown that, to some extent, the presence of dependencies between the syntactic parameters listed in the SSWL database can be detected using Kanerva networks. The latter were introduced in [16] as sparse distributed memories aimed at modeling associative memory in neuroscience. It is well known that, in fact, Kanerva networks are very useful for reconstructing corrupted data and detecting the degree of recoverability of certain parts of the data as a function of the remaining ones. In particular, this makes them suitable for detecting the presence of relations between data. It was shown in [26] that different syntactic parameters in the SSWL database exhibit different degrees of recoverability in a Kanerva network. An overall effect can be identified, which depends on the frequency with which a certain syntactic parameter is expressed across world languages. This effect can be reproduced using random data with the same frequencies. However, 
there is an additional effect that can be detected normalizing with respect to the frequency and that should be a genuine expression of the level of dependence of a particular syntactic parameter upon the remaining ones. The resulting normalized score computed in [26] is therefore a numerical estimate of the degree of dependence/independence of a given binary syntactic variable. The presence of these computationally detectable dependence relations affects some of the fundamental assumptions of the Markov models of phylogenetic trees, in particular the assumption that all the binary variables are independent, identically distributed variables. A possible way to compensate for this problem in the model it to consider a weighted version of the joint probability distribution $P=p_{i_{1} \ldots, i_{n}}$ at the leaves of the phylogenetic tree, where the frequency of expression of the parameters is computed in such a way that each parameter is weighted according to the corresponding normalized degree of recoverability in a Kanerva network, in such a way that the independent variables are weighted more than the dependent ones. This restores the fact that the independent variables assumption of the Markov model can be at least approximately satisfied.

\section{APPENDIX: THE SSWL PARAMETERS OF THE LATIN LANGUAGES}

The phylogenetic invariants for the tree of Latin languages of Figure 11 are evaluated at the probability distribution $p_{i_{1}, i_{2}, i_{3}, i_{4}, i_{5}}$ at the leaves, based on the SSWL parameters for this group of languages. There are 106 parameters in the SSWL database that are completely mapped for all of these five languages. We have excluded from the list all those SSWL parameters that are only mapped for some but not all of the languages in this group. With the notation $\ell_{1}=$ French, $\ell_{2}=$ Italian, $\ell_{3}=$ Latin, $\ell_{4}=$ Spanish, and $\ell_{5}=$ Portuguese, the syntactic parameters are given by the following list. The column on the left lists the SSWL parameters $P$ as labeled in the database, 35 .

\begin{tabular}{|l||c|}
\hline $\mathrm{P}$ & {$\left[\ell_{1}, \ell_{2}, \ell_{3}, \ell_{4}, \ell_{5}\right]$} \\
\hline 01 & {$[1,1,1,1,1]$} \\
02 & {$[0,1,1,1,1]$} \\
03 & {$[1,1,1,1,1]$} \\
04 & {$[0,0,1,0,0]$} \\
05 & {$[1,1,1,1,1]$} \\
06 & {$[0,0,1,0,0]$} \\
07 & {$[0,0,1,0,0]$} \\
08 & {$[0,0,1,0,0]$} \\
09 & {$[0,0,1,0,0]$} \\
10 & {$[0,0,1,0,0]$} \\
11 & {$[1,1,1,1,1]$} \\
12 & {$[0,0,1,0,0]$} \\
13 & {$[1,1,1,1,1]$} \\
14 & {$[1,1,1,1,1]$} \\
15 & {$[1,1,1,1,1]$} \\
16 & {$[0,0,1,0,0]$} \\
17 & {$[1,1,1,1,1]$} \\
18 & {$[0,0,1,0,0]$} \\
19 & {$[0,0,1,0,0]$} \\
20 & {$[1,1,1,1,1]$} \\
21 & {$[1,1,1,1,1]$} \\
22 & {$[0,0,1,1,1]$} \\
A01 & {$[1,1,1,1,1]$} \\
A02 & {$[1,1,1,1,1]$} \\
A03 & {$[1,1,1,1,1]$} \\
A04 & {$[0,1,1,0,1]$} \\
Aux Sel 01 & {$[1,1,0,0,0]$} \\
C01 & {$[1,1,1,1,1]$} \\
C02 & {$[0,0,0,0,0]$} \\
C03 & {$[1,1,1,1,1]$} \\
C04 & {$[0,0,0,0,0]$} \\
EE & {$[1,1,0,1,0]$} \\
\hline
\end{tabular}

\begin{tabular}{|l||l|}
\hline N2 01 & {$[1,1,1,1,1]$} \\
N2 02 & {$[0,0,1,0,0]$} \\
N2 03 & {$[1,1,0,1,1]$} \\
N2 04 & {$[0,0,1,0,0]$} \\
N2 05 & {$[1,1,0,1,1]$} \\
N2 06 & {$[1,1,1,1,1]$} \\
N2 07 & {$[0,0,0,0,0]$} \\
N2 08 & {$[0,0,0,0,0]$} \\
N2 09 & {$[0,0,0,0,0]$} \\
N2 10 & {$[0,0,0,0,0]$} \\
N2 11 & {$[0,0,0,0,0]$} \\
Neg 01 & {$[1,1,1,1,1]$} \\
Neg 02 & {$[1,0,0,0,0]$} \\
Neg 03 & {$[0,0,0,0,0]$} \\
Neg 04 & {$[0,0,0,0,0]$} \\
Neg 05 & {$[0,0,0,0,0]$} \\
Neg 06 & {$[0,0,0,0,0]$} \\
Neg 07 & {$[0,0,0,0,0]$} \\
Neg 08 & {$[0,0,0,0,0]$} \\
Neg 09 & {$[0,0,0,0,0]$} \\
Neg 10 & {$[0,0,0,0,0]$} \\
Neg 11 & {$[0,0,0,0,0]$} \\
Neg 12 & {$[0,0,0,0,0]$} \\
Neg 13 & {$[0,0,0,0,0]$} \\
Neg 14 & {$[0,0,0,0,0]$} \\
\hline
\end{tabular}

\begin{tabular}{|l||l|}
\hline Order N3 01 & {$[1,1,1,1,1]$} \\
Order N3 02 & {$[1,1,1,1,1]$} \\
Order N3 03 & {$[0,0,0,0,0]$} \\
Order N3 04 & {$[0,0,1,0,0]$} \\
Order N3 05 & {$[0,0,1,0,0]$} \\
Order N3 07 & {$[1,1,1,1,1]$} \\
Order N3 08 & {$[0,0,1,0,0]$} \\
Order N3 11 & {$[0,0,1,0,0]$} \\
Q01 & {$[1,0,0,0,0]$} \\
Q02 & {$[0,0,0,0,0]$} \\
Q03 & {$[0,0,1,0,0]$} \\
Q04 & {$[1,1,0,1,1]$} \\
Q05 & {$[1,0,0,1,0]$} \\
Q06 & {$[1,0,0,1,0]$} \\
Q07 & {$[0,0,0,0,0]$} \\
Q08 & {$[1,1,0,1,1]$} \\
Q09 & {$[0,0,1,0,1]$} \\
Q10 & {$[0,0,0,0,1]$} \\
Q11 & {$[0,0,0,0,0]$} \\
Q12 & {$[0,0,0,0,0]$} \\
Q13 & {$[0,0,0,0,0]$} \\
Q14 & {$[0,0,1,0,1]$} \\
Q15 & {$[0,0,0,0,0]$} \\
Q16 & {$[1,1,0,0,0]$} \\
Q17 & {$[0,0,1,1,1]$} \\
Q18 & {$[0,0,1,0,1]$} \\
Q21 & {$[1,0,0,0,0]$} \\
Q22 & {$[0,0,0,0,0]$} \\
\hline
\end{tabular}

\begin{tabular}{|l|l|}
\hline V2 01 & {$[0,0,0,0,0]$} \\
V2 02 & {$[0,0,0,1,0]$} \\
w01a & {$[0,1,1,1,1]$} \\
w01b & {$[1,0,0,0,0]$} \\
w01c & {$[0,1,0,0,0]$} \\
w02a & {$[0,0,1,0,0]$} \\
w02b & {$[1,1,0,1,1]$} \\
w02c & {$[0,0,0,0,0]$} \\
w03a & {$[0,0,1,0,0]$} \\
w03b & {$[1,1,0,1,1]$} \\
w03c & {$[0,0,0,0,0]$} \\
w03d & {$[0,0,0,0,0]$} \\
w04a & {$[0,0,1,0,0]$} \\
w04b & {$[1,1,0,1,1]$} \\
w04c & {$[0,0,1,0,0]$} \\
w05a & {$[0,1,1,1,1]$} \\
w05b & {$[1,0,0,0,0]$} \\
w05c & {$[0,1,0,1,1]$} \\
w06a & {$[0,0,1,0,0]$} \\
w06b & {$[1,1,0,1,1]$} \\
w06c & {$[0,0,1,0,0]$} \\
\hline
\end{tabular}


One can see by inspecting the different groups of parameters in this list that several parameters within the "same group" tend to behave in the same way (e.g. all the Neg parameters) or in more highly correlated way than across groups of parameters. This observation is consistent with the more general observation of dependencies observed through the Kanerva networks method in [26]. Thus, in order to better fit this set of binary variables with the hypothesis of independent equally distributed variables in Markov processes, it may be better to select a subset of the SSWL parameters that cuts across the various groups of more closely correlated variables. We will discuss this aspect more in details elsewhere.

The probability $p_{i_{1}, i_{2} . i_{3}, i_{4}, i_{5}}$ is then computed by counting the frequencies of occurrence of binary vectors $\left[i_{1}, i_{2}, i_{3}, i_{4}, i_{5}\right] \in\{0,1\}^{5}$ among the 106 vectors of SSWL parameters above. The only nonzero frequencies are

$$
\begin{array}{llll}
p_{0,0,0,0,0}=\frac{31}{106}, & p_{0,0,0,0,1}=\frac{1}{106}, & p_{0,0,0,1,0}=\frac{1}{106}, & p_{0,0,1,0,0}=\frac{23}{106}, \\
p_{0,0,1,0,1}=\frac{3}{106}, & p_{0,0,1,1,1}=\frac{2}{106}, & p_{0,1,0,0,0}=\frac{1}{106}, & p_{0,1,0,1,1}=\frac{1}{106}, \\
p_{0,1,1,0,1}=\frac{1}{106}, & p_{0,1,1,1,1}=\frac{3}{106}, & p_{1,0,0,0,0}=\frac{5}{106}, & p_{1,0,0,1,0}=\frac{2}{106}, \\
p_{1,1,0,1,0}=\frac{1}{106}, & p_{1,1,0,0,0}=\frac{2}{106}, & p_{1,1,0,1,1}=\frac{8}{106}, & p_{1,1,1,1,1}=\frac{21}{106} .
\end{array}
$$

Note how these frequencies confirm some well known facts about the Latin languages. Syntactic parameters (as recorded in SSWL) are very likely to have remained the same across all five languages in the family, with a higher probability of a feature not allowed in Latin remaining not allowed in the other languages (31/106) than of a feature allowed in Latin remaining allowed in the other languages $(21 / 106)$. It is also very likely that a feature is the same in all the modern ones but different from Latin, with a much higher incidence of cases of a feature allowed in Latin becoming disallowed in all the other languages (23/106) than the other way around (8/106). Among the remaining possibilities, we see incidences where French has an allowed feature that is missing in the other languages (5/106) of disallowed (3/106) and cases where Latin and Portuguese have the same feature allowed, which is disallowed in the other languages (3/106): all other nonzero entries have only two or less occurrences. The resulting matrices for the edge flattenings of the tree of Figure 11 are then as computed in $\$ 5$.

\section{REFERENCES}

[1] E. Allman, J. Rhodes, Phylogenetic ideals and varieties for general Markov models, Adv. Appl. Math. Vol.40 (2008) 127-148.

[2] M. Baker, The Atoms of Language, Basic Books, 2001.

[3] F. Barbançon, S.N. Evans, L. Nakhleh, D. Ringe, T. Warnow, An experimental study comparing linguistic phylogenetic reconstruction methods, Diachronica, Vol.30 (2013) N.2, 143-170.

[4] C. Bocci, Topics in phylogenetic algebraic geometry, Expo. Math. 25 (2007) 235-259.

[5] A. Bouchard-Côté, D. Hall, T.L. Griffiths, D. Klein, Automated reconstruction of ancient languages using probabilistic models of sound change, Proceedings of the National Academy of Sciences (PNAS) vol.110 (2013) N.11, 4224-4229.

[6] R. Bouckaert, P. Lemey, M. Dunn, S.J. Greenhill, A.V. Alekseyenko, A.J. Drummond, R.D. Gray, M.A. Suchard, Q.D. Atkinson, Mapping the origins and expansion of the Indo-European language family, Science, Vol.337 (2012) 957-960.

[7] N. Chomsky, Lectures on Government and Binding, Dordrecht: Foris Publications, 1982.

[8] N. Chomsky, H. Lasnik, The theory of Principles and Parameters, in "Syntax: An international handbook of contemporary research", pp.506-569, de Gruyter, 1993. 
[9] M. DeGiorgio, J.H. Degnan, Robustness to divergence time underestimation when inferring species trees from estimated gene trees, Syst. Biol. Vol.63 (2014) N.1, 66-82.

[10] A. Delmestri, N. Cristianini, Linguistic phylogenetic inference by PAM-like matrices, Journal of Quantitative Linguistics 19 (2012) 95-120.

[11] N. Eriksson, K. Ranestad, B. Sturmfels, S. Sullivant, Phylogenetic Algebraic Geometry, in "Projective varieties with unexpected properties", pp.237-255, Walter de Gruyter, 2005.

[12] P. Forster, C. Renfrew, Phylogenetic methods and the prehistory of language, McDonald Institute Monographs, 2006.

[13] C. Galves (Ed.) Parameter Theory and Linguistic Change, Oxford University Press, 2012.

[14] D. Gusfield, ReCombinatorics, MIT Press, 2014.

[15] D.H. Huson, R. Rupp, C. Scornavacca, Phylogenetic Networks: Concepts, Algorithms and Applications, Cambridge University Press, 2010.

[16] P. Kanerva, Sparse Distributed Memory, MIT Press, 1988.

[17] G. Longobardi, Methods in parametric linguistics and cognitive history, Linguistic Variation Yearbook, Vol.3 (2003) 101-138.

[18] G. Longobardi, L. Bortolussi, M.A. Irimia, N. Radkevich, A. Ceolin, C. Guadagno, D. Michelioudakis, A. Sgarro, Mathematical modeling of grammatical diversity supports the historical reality of formal syntax, in "Proceedings of the Leiden Workshop on Capturing Phylogenetic Algorithms for Linguistics", 2016.

[19] G. Longobardi, S. Ghirotto, C. Guardiano, F. Tassi, A. Benazzo, A. Ceolin, G. Barbujani, Across language families: genome diversity mirrors linguistic variation within Europe, Am. J. Phys. Anthropol. Vol.157(2015) N.4, 630-640.

[20] G. Longobardi, C. Guardiano, Evidence for syntax as a signal of historical relatedness, Lingua, 119 (2009) 1679-1706.

[21] G. Longobardi, C. Guardiano, G. Silvestri, A. Boattini, A. Ceolin, Towards a syntactic phylogeny of modern Indo-European languages, Journal of Historical Linguistics, Vol.3 (2013) N.1, 122-152.

[22] M. Marcolli, Syntactic parameters and a coding theory perspective on entropy and complexity of language families, Entropy, Vol.18 (2016) 110 [17 pages]

[23] L. Nakhleh, D. Ringe, T. Warnow, Perfect phylogenetic networks: a new methodology for reconstructing the evolutionary history of natural languages, Language Vol. 81 (2005) N.2, 382-420.

[24] L. Pacher, B. Sturmfels, The Mathematics of Phylogenomics, SIAM Review, Vol.49 (2007) N.1, 3-31.

[25] L. Pacher, B. Sturmfels, Tropical geometry of statistical models, Proceedings of the National Academy of Sciences (PNAS) vol.101 (2004) N.46, 16132-16137.

[26] J.J.Park, R.Boettcher, A.Zhao, A.Mun, K.Yuh, V.Kumar, M.Marcolli, Prevalence and recoverability of syntactic parameters in sparse distributed memories, arXiv:1510.06342 [cs.CL]

[27] A. Perelysvaig, M.W. Lewis, The Indo-European controversy: facts and fallacies in Historical Linguistics, Cambridge University Press, 2015.

[28] F. Petroni, M. Serva, Language distance and tree reconstruction, J. Stat. Mech. (2008) P08012 [16 pages]

[29] A. Port, I. Gheorghita, D. Guth, J.M. Clark, C. Liang, S. Dasu, M. Marcolli, Persistent Topology of Syntax, arXiv:1507.05134 [cs.CL]

[30] L. Rizzi, On the format and locus of parameters: the role of morphosyntactic features, preprint, 2016.

[31] N. Saitou, M. Nei, The Neighbor-joining Method: a new method for reconstructing phylogenetic trees, Mol. Biol. Evol. Vol.4 (1987) N.4, 406-425.

[32] K. Siva, J. Tao, M. Marcolli, Spin glass models of syntax and language evolution, arXiv:1508.00504 [cs.CL]

[33] B. Sturmfels, S. Sullivant, Toric ideals of phylogenetic invariants, J. Comput. Bio. 12 (2005) N.2, 204-228.

[34] T. Warnow, S.N. Evans, D. Ringe, L. Nakhleh, Stochastic models of language evolution and an application to the Indo-European family of languages, available at http://www.stat.berkeley.edu/users/evans/659.pdf

[35] SSWL Database of Syntactic Parameters: http://sswl.railsplayground.net/ 
Division of Physics, Mathematics, and Astronomy, California Institute of Technology, 1200 E. California Blvd, Pasadena, CA 91125, USA

E-mail address: kshu@caltech.edu

E-mail address: saziz@caltech.edu

E-mail address: vhuynh@caltech.edu

E-mail address: warrick.david58@gmail.com

E-mail address: matilde@caltech.edu 